\title{
Natural Philosophy and Human Cultures*
}

\author{
By Prof. Niels Bohr, For. Mem. R.S.
}

$\mathrm{I}^{\mathrm{T}}$ $T$ is only with great hesitation that I have accepted a kind invitation to address this assembly of distinguished representatives of the anthropological and ethnographical sciences of which I, as a physicist, have of course no first-hand knowledge. Still, on this special occasion, when even the historical surroundings speak to everyone of us about aspects of life other than those discussed at the regular congress proceedings, it might perhaps be of interest to try with a few words to direct attention to the epistemological aspect of the latest development of natural philosophy and its bearing on general human problems. Notwithstanding the great separation between our different branches of knowledge, the new lesson which has been impressed upon physicists regarding the caution with which all usual conventions must be applied as soon as we are not concerned with everyday experience, may, indeed, be suited to remind us in a novel way of the dangers, well known to humanists, of judging from our own point of view cultures developed within other societies.

Of course it is impossible to distinguish sharply between natural philosophy and human culture. The physical sciences are, in fact, an integral part of our civilization, not only because our ever increasing mastery of the forces of Nature has so completely changed the material conditions of life, but also because the study of these sciences has contributed so much to clarify the background of our own existence. What has it not meant in this respect, that we no more consider ourselves as privileged in living at the centre of the universe, surrounded by less fortunate societies inhabiting the edges of the abyss, but that through the development of astronomy and geography we have realized that we are all sharing a small spherical planet of the solar system, which again is only a small part of still larger systems. How forceful an admonition about the relativity of all human judgements have we not also in our days received through the renewed revision of the presuppositions underlying the unambiguous use of even our most elementary concepts such as space and time, which, in disclosing the essential dependence of every physical phenomenon on the point of view of the observer, has contributed so largely to the unity and beauty of our whole world-picture.

While the importance of these great achievements

* Address given at Kronborg Castle during the excursion to Elsinore of the International Congress of Anthropological and Ethnologica Sciences, Copenhagen, August, 1938 for our general outlook is commonly realized, it is scarcely yet so as regards the unsuspected epistemological lesson which the opening of quite new realms of physical research has given us in the most recent years. Our penetration into the world of atoms, hitherto closed to the eyes of man, is indeed an adventure which may be compared with the great journeys of discovery of the circumnavigators and the bold explorations of astronomers into the depths of celestial space. As is well known, the marvellous development of the art of physical experimentation has not only removed the last traces of the old belief that the coarseness of our senses would for ever prevent us from obtaining direct information about individual atoms, but has even shown us that the atoms themselves consist of still smaller corpuscles which can be isolated and the properties of which can be investigated separately. At the same time we have, however, in this fascinating field of experience been taught that the laws of Nature hitherto known, which constitute the grand edifice of classical physics, are only valid when dealing with bodies consisting of practically infinite numbers of atoms. The new knowledge concerning the behaviour of single atoms and atomic corpuscles has, in fact, revealed an unexpected limit for the subdivision of all physical actions extending far beyond the old doctrine of the limited divisibility of matter, and giving every atomic process a peculiar individual character. This discovery has, in fact, yielded a quite new basis for the understanding of the intrinsic stability of atomic structures, which, in the last resort, conditions the regularities of all ordinary experience.

How radical a change in our attitude towards the description of Nature this development of atomic physics has brought about is perhaps most clearly illustrated by the fact that even the principle of causality, so far considered as the unquestioned foundation for all interpretation of natural phenomena, has proved too narrow a frame to embrace the peculiar regularities governing individual atomic processes. Certainly everyone will understand that physicists have needed very cogent reasons to renounce the ideal of causality itself; but in the study of atomic phenomena we have repeatedly been taught that questions which were believed to have received long ago their final answers had most unexpected surprises in store for us. You will surely all have heard. about the riddles regarding the most elementary 
properties of light and matter which have puzzled physicists so much in recent years. The apparent contradictions which we have met in this respect are, in fact, as acute as those which gave rise to the development of the theory of relativity in the beginning of this century, and have, just as the latter, only found their explanation by a closer examination of the limitation imposed by the new experiences themselves on the unambiguous use of the concepts entering into the description of the phenomena. While in relativity theory the decisive point was the recognition of the essentially different ways in which observers moving relatively to each other will describe the behaviour of given objects, the elucidation of the paradoxes of atomic physics has disclosed the fact that the unavoidable interaction between the objects and the measuring instruments sets an absolute limit to the possibility of speaking of a behaviour of atomic objects which is independent of the means of observation.

We are here faced with an epistemological problem quite new in natural philosophy, where all description of experiences has so far been based upon the assumption, already inherent in ordinary conventions of language, that it is possible to distinguish sharply between the behaviour of objects and the means of observation. This assumption is not only fully justified by all everyday experience, but even constitutes the whole basis of classical physics, which, just through the theory of relativity, has received such a wonderful completion. As soon as we begin to deal, however, with phenomena like individual atomic processes which, due to their very nature, are essentially determined by the interaction between the objects in question and the measuring instruments necessary for the definition of the experimental arrangements, we are forced to examine more closely the question of what kind of knowledge can be obtained concerning these objects. In this respect we must, on one hand, realize that the aim of every physical experiment-to gain knowledge under reproducible and communicable conditions-leaves us no choice but to use everyday concepts, eventually refined by the terminology of classical physics, not only in all accounts of the construction and manipulation of the measuring instruments but also in the description of the actual experimental results. On the other hand, it is equally important to understand that just this circumstance implies that no result of an experiment concerning a phenomenon which, in principle, lies outside the range of classical physics, can be interpreted as giving information about independent properties of the object; but is inherently connected with a definite situation in the description of which the measuring instruments interacting with the objects also enter essentially. This last fact gives the straightforward explanation of the apparent contradictions which appear when results about atomic objects obtained by different experimental arrangements are tentatively com. bined into a self-contained picture of the object.

Information regarding the behaviour of an atomic object obtained under definite experimental conditions, may, however, according to a terminology often used in atomic physics, be adequately characterized as complementary to any information about the same object obtained by some other experimental arrangement excluding the fulfilment of the first conditions. Although such kinds of information cannot be combined into a single picture by means of ordinary concepts, they represent indeed equally essential aspects of any knowledge of the object in question which can be obtained in this domain. It is the recognition of such a complementary character of the mechanical analogies by which one has tried to visualize the individual radiative effects which has, in fact, led to an entirely satisfactory solution of the riddles of the properties of light alluded to above. In the same way, it is only by taking into consideration the complementary relationship between the different experiences concerning the behaviour of atomic corpusoles, that it has been possible to obtain a clue to the understanding of the striking contrast between the properties of ordinary mechanical models and the peculiar laws of stability governing atomic structures which form the basis for every closer explanation of the specific physical and chemical properties of matter.

Of course I have no intention, on this occasion, of entering more closely into such details, but I hope that I have been able to give a sufficiently clear impression of the fact that we are here in no way concerned with an arbitrary renunciation as regards the detailed analysis of the almost overwhelming richness of our rapidly increasing experience in the realm of atoms. On the contrary, we have to do with a rational development of our means of classifying and comprehending new experience which, due to its very character, finds no place within the frame of causal description suited only to account for the behaviour of objects so long as this behaviour is independent of the means of observation. Far from containing any mysticism contrary to the spirit of science, the viewpoint of 'complementarity' forms indeed a consistent generalization of the ideal of causality.

However unexpected this development may appear in the domain of physics, I am sure that many of you will have recognized the close analogy between the situation as regards the analysis of atomic phenomena, which I have described, and characteristic features of the problem of observation 
in human psychology. Indeed, we may say that the trend of modern psychology can be characterized as a reaction against the attempt at analysing psychical experience into elements which can be associated in the same way as are the results of measurements in classical physics. In introspection, it is clearly impossible to distinguish sharply between the phenomena themselves and their conscious perception, and although we may often speak of lending our attention to some particular aspect of a psychical experience, it will appear on closer examination that we really have to do, in such cases, with mutually exclusive situations. We all know the old saying that, if we try to analyse our own emotions, we scarcely possess them any longer, and in that sense we recognize between psychical experiences, for the description of which words such as 'thoughts' and 'feelings' are adequately used, a complementary relationship similar to that between the experiences regarding the behaviour of atoms obtained under different experimental arrangements and described by means of different analogies taken from our usual ideas. By such a comparison it is, of course, in no way intended to suggest any closer relation between atomic physics and psychology, but merely to stress an epistemological argument common to both fields, and thus to encourage us to see how far the solution of the relatively simple physical problems may be helpful in clarifying the more intricate psychological questions with which human life confronts us, and which anthropologists and ethnologists so often meet in their investigations.

Coming now closer to our subject of the bearing of such viewpoints on the comparison of different human cultures, we shall first stress the typical complementary relationship between the modes of behaviour of living beings characterized by the words 'instinct' and 'reason'. It is true that these words are used in very different senses; thus instinct may mean motive power or inherited behaviour, and reason may denote deeper sense as well as conscious argumentation. What we are concerned with is, however, only the practical way in which these words are used to discriminate between the different situations in which animals and men find themselves. Of course, nobody will deny our belonging to the animal world, and it would even be very difficult to find an exhaustive definition characterizing man among the other animals. Indeed, the latent possibilities in any living organism are not easily estimated, and I think that there is none of us who has not sometimes been deeply impressed by the extent to which circus animals can be drilled. Not even with respect to the conveyance of information from one individual to another would it be possible to draw a sharp separation between animals and man; but of course our power of speech places us in this respect in an essentially different situation, not only as regards the exchange of practical experience, but also and above all as regards the possibility of transmitting through education to children the traditions concerning behaviour and reasoning which form the basis of any human culture.

As regards reason compared with instinct, it is, above all, essential to realize that no proper human thinking is imaginable without the use of concepts framed in some language which every generation has to learn anew. This use of concepts is, in fact, not only to a large extent suppressing instinctive life, but stands even largely in an exclusive relationship of complementarity to the display of inherited instincts. The astonishing superiority of lower animals compared with man in utilizing the possibilities of Nature for the maintenance and propagation of life has certainly often its true explanation in the fact that, for such animals, we cannot speak of any conscious thinking in our sense of the word. At the same time, the amazing capacity of so-called primitive people to orientate themselves in forests or deserts, which, though apparently lost in more civilized societies, may on occasion be revived in any of us, might justify the conclusion that such feats are only possible when no recourse is taken to conceptual thinking, which on its side is adapted to far more varied purposes of primary importance for the development of civilization. Just because it is not yet awake to the use of concepts, a new-born child can scarcely be reckoned as a human being; but belonging to the species of man, it has, of course, though more helpless a creature than most young animals, the organic possibilities of receiving through education a culture which enables it to take its place in some human society.

Such considerations confront us at once with the question whether the widespread belief that every child is born with a predisposition for the adoption of a specific human culture is really well-founded, or whether one has not rather to assume that any culture can be implanted and thrive on quite different physical backgrounds. Here we are of course touching a subject of still unsettled controversies between geneticists, who pursue most interesting studies on the inheritance of physical characters. In connexion with such discussions, however, we must above all bear in mind that the distinction between the concepts genotype and phenotype, so fruitful for the clarification of heredity in plants and animals, essentially presupposes the subordinate influence of the external conditions of life on the characteristic properties of the species. In the case of the specific cultural 
characters of human societies the problem is, however, reversed in the sense that the basis for the classification is here the traditional habits shaped by the histories of the societies and their natural environments. These habits, as well as their inherent presuppositions, must therefore be analysed in detail before any possible influence of inherited biological differences on the development and maintenance of the cultures concerned can be estimated. Indeed, in characterizing different nations and even different families within a nation, we may to a large extent consider anthropological traits and spiritual traditions as independent of each other, and it would even be tempting to reserve by definition the adjective 'human' for just those characters which are not directly bound to bodily inheritance.

At first sight, it might perhaps appear that such an attitude would mean unduly stressing merely dialectic points. But the lesson which we have received from the whole growth of the physical sciences is that the germ of fruitful development often lies just in the proper choice of definitions. When we think, for example, of the clarification brought about in various branches of science by the argumentation of relativity theory, we see indeed what advance may lie in such formal refinements. As I have already hinted earlier in this address, relativistic viewpoints are certainly also helpful in promoting a more objective attitude as to relationships between human cultures, the traditional differences of which in many ways resemble the different equivalent modes in which physical experience can be described. Still, this analogy between physical and humanistic problems is of limited scope and its exaggeration has even led to misunderstandings of the essence of the theory of relativity itself. The unity of the relativistic world picture, in fact, implies the possibility for any one observer to predict within his own conceptual frame how any other observer will co-ordinate experience within the frame natural to him. The main obstacle to an unprejudiced attitude towards the relation between various human cultures is, however, the deep-rooted differences of the traditional backgrounds on which the cultural harmony in different human societies is based and which exclude any simple comparison between such cultures.

It is above all in this connexion that the viewpoint of complementarity offers itself as a means of coping with the situation. In fact, when studying human cultures different from our own, we have to deal with a particular problem of observation which on closer consideration shows many features in common with atomic or psychological problems, where the interaction between objects and measuring tools, or the inseparability of objective content and observing subject, prevents an immediate application of the conventions suited to accounting for experiences of daily life. Especially in the study of cultures of primitive peoples, ethnologists are, indeed, not only aware of the risk of corrupting such cultures by the necessary contact, but are even confronted with the problem of the reaction of such studies on their own human attitude. What I here allude to is the experience, well known to explorers, of the shaking of their hitherto unrealized prejudices through the experience of the unsuspected inner harmony human life can present even under conventions and traditions most radically different from their own. As a specially drastic example I may perhaps here recall the extent to which in certain societies the roles of men and women are reversed, not only regarding domestic and social duties but also regarding behaviour and mentality. Even if many of us, in such a situation, might perhaps at first shrink from admitting the possibility that it is entirely a caprice of fate that the people concerned have their specific culture and not ours, and we not theirs instead of our own, it is clear that even the slightest suspicion in this respect implies a betrayal of the national complacency inherent in any human culture resting in itself.

Using the word much as it is used, in atomic physics, to characterize the relationship between experiences obtained by different experimental arrangements and capable of visualization only by mutually exclusive ideas, we may truly say that different human cultures are complementary to each other. Indeed, each such culture represents a harmonious balance of traditional conventions by means of which latent potentialities of human life can unfold themselves in a way which reveals to us new aspects of its unlimited richness and variety. Of course, there cannot, in this domain, be any question of such absolutely exclusive relationships as those between complementary experiences about the behaviour of well-defined atomic objects, since scarcely any culture exists which could be said to be fully self-contained. On the contrary, it is well known from numerous examples how a more or less intimate contact between different human societies can lead to a gradual fusion of traditions, giving birth to a quite new culture. The importance in this respect of the mixing of populations through emigration or conquest for the advancement of human civilization need scarcely be recalled. It is, indeed, perhaps the greatest prospect of humanistic studies, to contribute through an increasing knowledge of the history of cultural development to that gradual removal of prejudices which is the common aim of all science. 
As I stressed in the beginning of this address, it is, of course, far beyond my capacities to contribute in any direct way to the solution of the problems discussed among the experts at this congress. My only purpose has been to give an impression of a general epistemological attitude which we have been forced to adopt in a field as far from human passions as the analysis of simple physical experiments. I do not know, however, whether I have found the right words to convey this impression, and before I conclude, I may perhaps be allowed to relate an experience which once most vividly reminded me of my deficiencies in this respect. In order to explain to an audience that I did not use the word 'prejudice' to imply any condemnation of other cultures, but merely to characterize our necessarily prejudiced con- ceptual frame, I referred jokingly to the traditional prejudices which the Danes eherish with regard to their Swedish brothers on the other side of the beautiful Sound outside these windows, with whom we have fought through centuries even within the walls of this castle, and from contact with whom we have, through the ages, received so much fruitful inspiration. Now you will realize what a shock I got when, after my address, a member of the audience came up to me and said that he could not understand why I hated the Swedes. Obviously I must have expressed myself most confusingly on that occasion, and I am afraid that also to-day I have talked in a very obscure way. Still, I hope that I have not spoken so obscurely as to give rise to any such misunderstandings of the trend of my argument.

\section{Modern Trends in Air Transport*}

$\mathrm{M}^{\mathrm{os}}$ OST modern air transport is by means of the aeroplane, a body heavier than air, depending upon forward movement for sustentation. For purposes of this examination it can be considered to consist of four fundamentally different systems : (1) the lifting system, that is, the wings ; (2) the non-lifting system, principally the body, a structure with space for the load, a girder carrying the tail control units, and often the power plant; (3) the propulsive system comprising the engines and airscrews; (4) the control system, including all devices by which the pilot governs the movements of his aircraft. The trend in development of each of these is examined from the point of view of its approach towards air transport ideals of carrying capacity, speed, range, economy, comfort and safety.

The lift of a wing increases with its area, the square of the speed, and, in general, its angle of attack. Increase of lift therefore demands some combination of larger values of these. It must be remembered that carrying capacity is not gross lift; but rather the surplus of the total lift over that necessary to sustain the weight of the structure, engines, etc. Assuming wing structures carrying the same load per unit area, with the same geometry of structure and materials of construction, while the lift will increase as the square of the dimensions, the volume, and consequently the weight, of the wing will increase as

* Based on a lecture by Prof. W. F. Durand, emeritus professor of mechanical engineering in Stanford University, given at the recent meeting of the American Association for the Advancement of Science, Richmond, Virginia. the cube of these. This relationship, known as the square/cube law, implies that with weight increasing at a greater rate than lift, the carrying capacity of any wing system will ultimately be reduced to zero. The functioning of this law is being defeated by designers by the more efficient distribution of the materials in the structure, the use of specifically stronger (strength/weight) materials, and improved aerodynamic design allowing increased speed for the same power, and consequently extra lift from the same wing area. This is further helped by the fact that the power required is a factor of the wing area, and its weight is only increasing as the square of the linear dimensions of the machine. Increase in angle of attack, as a method of obtain. ing additional lift, results in an increase in drag of the wing at such a rate that the weight of the extra power plant necessary to overcome it soon cancels out its extra lift. As an example, early post-War commercial aeroplanes weighed from three to five thousand pounds with a wing loading of eight to twelve pounds per square foot, and there was evidence that the optimum size under the square/cube law was already approaching. A new Boeing flying boat, now building, will weigh $82,000 \mathrm{lb}$. with a carrying capacity of $10,000 \mathrm{lb}$., while some projected designs for trans-ocean services are of $200,000 \mathrm{lb}$. total weight and expect to carry a pay load of $25,000 \mathrm{lb}$., with wing loadings of $30-40 \mathrm{lb}$. per square foot.

The question of speeds is one of commercial requirements. They have risen from 50-80 miles per hour to 150-200 miles per hour normal cruising 